\title{
Clinical connectome fingerprints of cognitive decline
}

\author{
Pierpaolo Sorrentino ${ }^{\mathrm{a}, 1}$, Rosaria Rucco ${ }^{\mathrm{b}, \mathrm{c}, 1}$, Anna Lardone ${ }^{\mathrm{d}}$, Marianna Liparoti ${ }^{\mathrm{c}}$, Emahnuel Troisi \\ Lopez $^{\mathrm{c}}$, Carlo Cavaliere ${ }^{\mathrm{e}}$, Andrea Soricelli ${ }^{\mathrm{c}, \mathrm{e}}$, Viktor Jirsa ${ }^{\mathrm{a}}$, Giuseppe Sorrentino ${ }^{\mathrm{b}, \mathrm{c}, \mathrm{f}, \mathrm{f},}$ \\ Enrico Amico ${ }^{g, h, * *}$ \\ ${ }^{a}$ Institut de Neurosciences des Systèmes, Aix-Marseille Université, Marseille, France \\ ${ }^{\mathrm{b}}$ Institute of Applied Sciences and Intelligent Systems, CNR, Pozzuoli, Italy

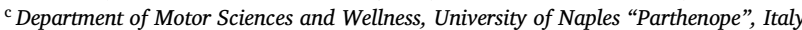 \\ ${ }^{\mathrm{d}}$ Department of Social and Developmental Psychology, University of Rome "Sapienza, Italy \\ e IRCCS SDN, Naples, Italy \\ ${ }^{\mathrm{f}}$ Hermitage Capodimonte Clinic, Naples, Italy \\ ${ }^{\mathrm{g}}$ Institute of Bioengineering, Center for Neuroprosthetics, EPFL, Geneva, Switzerland \\ ${ }^{\mathrm{h}}$ Department of Radiology and Medical Informatics, University of Geneva (UNIGE), Geneva, Switzerland
}

\section{A R T I C L E I N F O}

\section{Keywords:}

Clinical brain fingerprinting

Functional connectomes

Cognitive impairment

MEG connectivity

Brain networks

\begin{abstract}
A B S T R A C T
Brain connectome fingerprinting is rapidly rising as a novel influential field in brain network analysis. Yet, it is still unclear whether connectivity fingerprints could be effectively used for mapping and predicting disease progression from human brain data. We hypothesize that dysregulation of brain activity in disease would reflect in worse subject identification. We propose a novel framework, Clinical Connectome Fingerprinting, to detect individual connectome features from clinical populations. We show that "clinical fingerprints" can map individual variations between elderly healthy subjects and patients with mild cognitive impairment in functional connectomes extracted from magnetoencephalography data. We find that identifiability is reduced in patients as compared to controls, and show that these connectivity features are predictive of the individual Mini-Mental State Examination (MMSE) score in patients. We hope that the proposed methodology can help in bridging the gap between connectivity features and biomarkers of brain dysfunction in large-scale brain networks.
\end{abstract}

\section{Introduction}

Alzheimer's disease (AD) is the most common form of dementia worldwide. It is well known that the pathophysiological processes start years, and possibly decades, before the clinical onset (Braak and Braak, 1991). Consequently, the identification of subjects carrying a high risk of developing the disease is necessary to study the early stage of AD pathophysiology and to adopt new and more successful therapeutic approaches. This led to the definition of the clinical construct of mild cognitive impairment (MCI) (Petersen et al., 1999). According to the first conceptualization, MCI has been regarded as a clinical condition characterized by an objective memory impairment not yet encompassing the definition of dementia, but with a higher risk of developing severe cognitive decline (Petersen et al., 1999). Currently, MCI patients are classified according to type and number of affected cognitive domains. This clini- cal classification is particularly relevant because each subtype is linked to a presumed etiology, in fact the amnestic subtypes (aMCI) seems to represent the prodromal form of AD (Petersen et al., 1999).

Typically, the main symptom in aMCI is memory impairment. However, when this condition progresses toward the overt dementia phase, several cognitive functions become compromised, such as comprehension, communication, problem-solving, abstraction, imagining, planning, logic reasoning and abstract thought. To date, it has not been possible to link such functions to the malfunctioning of any specific area. This could be due to the fact that such complex abilities might not stem from a single dysfunctional area, but rather from the coordinated activity of multiple brain regions, which can be represented as a brain network or connectome (Bullmore and Sporns, 2009).

In brain networks, nodes correspond to gray-matter regions (based on brain atlases or parcellations), while links or edges correspond to

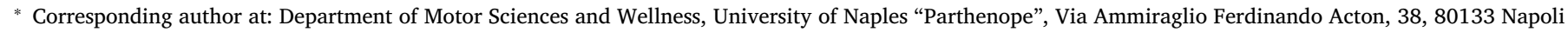
NA, Italy.

** Corresponding author at: Institute of Bioengineering, EPFL, Campus Biotech, Chemin des Mines 9, 1202 Genève.

E-mail addresses: giuseppe.sorrentino@uniparthenope.it (G. Sorrentino), enrico.amico@epfl.ch (E. Amico).

1 The authors contributed equally 
connections (either structural or functional) among them (Fornito et al., 2016). Recent advances in functional neuroimaging have provided new tools to measure these connections, by exploiting the statistical dependencies between brain signals, giving rise to the field of functional connectivity or functional brain connectomics (Friston, 2011). Examining functional connectivity in the human brain offers unique insights on how integration and segregation of information relates to human behavior and how this organization may be altered in diseases (Fornito et al., 2015). Indeed, considerable evidence has confirmed that anomalies in either the co-activations, the synchronization and/or the topology of the brain network are likely occurring in MCI (Contreras et al., 2017; Jacini et al., 2018).

Despite the progress made in this direction, two main problems have arisen when using brain network models as a way to detect functional connectivity alterations in AD and/or MCI. Firstly, the clinical interpretation became more challenging, and behavioral correlates necessary to interpret the findings. Secondly, lack of replicability hindered the generalization of the results (Botvinik-Nezer et al., 2020). Hence, despite considerable efforts from the community, reliably linking functional alterations to the MCI condition in a methodologically reliable and clinically valid way has been proven elusive (Engels et al., 2017). However, recent work on fMRI and EEG data showed that the individual connectivity does allow reliable single-subject identification in the healthy (Amico and Goñi, 2018; Finn et al., 2015; MirandaDominguez et al., 2014), given that good enough test-to-test reliability is provided (Noble et al., 2019). Nevertheless, the relationship between reliability ("connectivity fingerprinting") and validity (associations with disease-related biomarkers) still lacks definitive answers. In other words, how does connectivity fingerprinting relate to alterations in the diseased connectomes?

Here, we introduce a methodology to test for the reliability/validity relations in clinical populations, that we named Clinical Connectome Fingerprinting (CCF). The key distinction between CCF and "standard" connectome fingerprinting is that, in the CCF framework, we compare the similarity of the connectomes across test-retest sessions in patients against controls, obtaining individual similarity scores for each patient. We use these similarity scores as biomarkers for the prediction of clinical scores associated with the disease at hand. This idea is based on the consideration that the individual similarity scores obtained from CCF might provide a summary of large-scale dysregulation taking place in diseased brains. Starting from this assumption, we further hypothesised that individual alterations in the connectivity profiles, as summarized by CCF, might be associated with clinical outcomes of widespread cognitive decline, such as the Mini-Mental State Examination (MMSE).

We applied the CCF technique to source-reconstructed magnetoencephalographic (MEG) data in aMCI subjects and matched healthy subjects (HS). We started with comparing the identification performance of a variety of connectivity/synchronization metrics that are commonly used to derive functional connectomes from MEG data. We selected the best performing one for further analysis, namely the phase linearity measurement (PLM) (Baselice et al., 2019). We observed a consistent drop in connectome fingerprinting when transitioning from healthy to aMCI. Then, in order to test reliability/validity relationships in our dataset, we used intra-class correlation coefficient (ICC) to rank the edges according to their reliability in connectome fingerprinting. That is, we identified the edges that are more stable across test-retest sessions in controls, since the fingerprinting is mostly reliant on such edges. This analysis was carried out for different frequency bands separately, likely pinpointing specific circuitry (Jacini et al., 2018). We hypothesized that the same links responsible for the lack of identifiability in the MCI cohort would also be related to the symptomatology. Hence, we show that the most reliable links in the healthy (and whose reliability drops in the MCI population, as said) are indeed the ones predicting individual global cognitive impairment in patients, as measured by the Mini Mental State Examination (MMSE) scores.

\section{Materials and methods}

\subsection{Participants}

For this study, eighty-six patients referring to the Center for Cognitive and Memory Disorders of the Hermitage Capodimonte Clinic in Naples were consecutively recruited. All subjects, aged 53 to 81, were right handed (none of them had any left-handed relatives) and native Italian speakers. Thirty-four age- gender- body mass index (BMI)- and education- matched subjects among patients spouses or friends were enrolled as control group (HSs). Exclusion criteria were the presence of neurological or systemic illness that could affect the cognitive status, and contraindications to MRI or MEG recording.

Both patients and HS underwent the following screening: neurological examination, extensive neuropsychological assessment (see Table 1), MRI scan (including hippocampal volume evaluation) and MEG recording. MCI diagnosis was formulated according to the National Institute on Aging-Alzheimer Association (NIA-AA) criteria (Albert et al., 2011), which include: (i) cognitive concern reported by patient or informant or clinician, (ii) objective evidence of impairment in one or more cognitive domains, typically including memory, (iii) preservation of independence in functional abilities, (iv) not demented. Reduced hippocampal volume detected by structural MRI gives our aMCI cohort an intermediate likelihood of being due to AD (Albert et al., 2011).

Screened subjects with either MRI alterations (traumatic brain injury, meningioma, lacunar infarction), diagnosis of depression, dementia or non-amnestic MCI were excluded from further analysis. The subjects included in the study were 35 patients affected by aMCI (mean \pm SD age $71.20 \pm 6.67$ years; 18 men and 17 women) compared to 34 age, educational level and gender matched healthy subjects (mean \pm SD age $69.88 \pm 5.56$ years; 19 men and 15 women).

The study was approved by the Local Ethics Committee "Comitato Etico Campania Centro" (Prot.n.93C.E./Reg. n.14-17OSS), and all subjects gave written informed consent. All methods included in the protocol were carried out in accordance with the Declaration of Helsinki.

\subsection{Magnetic resonance imaging acquisition}

For $24 \mathrm{HS}$ and $32 \mathrm{MCI}$ patients, MR images were acquired using a 3T Biograph mMR tomograph (Siemens Healthcare, Erlangen, Germany) equipped with a 12 channels head coil. The scan was performed after the MEG registration or at least 21 days before (within 1 month). The MR registration protocol was: (i) three-dimensional T1weighted Magnetization-Prepared Rapid Acquisition Gradient-Echo sequence (MPRAGE, 240 sagittal planes, $214 \times 21 \mathrm{~mm} 2$ Field of View, voxel size $1 \times 1 \times 1 \mathrm{~mm} 3$, TR/TE/TI $2400 / 2.5 / 1000 \mathrm{~ms}$, flip angle $8^{\circ}$ ); (ii) Three-dimensional T2-weighted Sampling Perfection with Application optimized. Contrasts using different flip angle Evolution sequence (SPACE, 240 sagittal planes, $214 \times 214 \mathrm{~mm} 2$ Field of View, voxel size $1 \times 1 \times 1 \mathrm{~mm} 3$, TR/TE 3370/563); (iii) Two-dimensional T2- weighted turbo spin echo Fluid Attenuated Inversion Recovery sequence (FLAIR, 44 axial planes, $230 \times 230 \mathrm{~mm} 2$ Field of View, voxel size $0.9 \times 0.9 \times 0.9 \mathrm{~mm} 3$, TR/TE/TI $9000 / 95 / 25,00$, flip angle $150^{\circ}$ ).

The volumetric analysis was performed using the Freesurfer software (version 6.0) (FreeSurfer, 2012), specifically the normalization of the volumes was made by the estimated total intracranial volume (eTIV) while the Fazekas scale was used to evaluate the vascular burden (Fazekas et al., 1987). For the remaining participants who refused or did not complete the MR scan we used a standard MRI model.

\section{3. $M E G$ acquisition and preprocessing}

The data were acquired using a MEG system equipped by 163 magnetometers SQUID (Superconducting Quantum Interference Device), developed by the National Research Council of Italy at the Institute of Ap- 
Table 1

Neuropsychological evaluation.

\begin{tabular}{ll}
\hline Test & Explored function \\
\hline MMSE & $\begin{array}{l}\text { Global cognitive status } \\
\text { FAB }\end{array}$ \\
BDI & Depression \\
MDB & Short and long-term verbal episodic memory \\
Rey's 15 word immediate recall & Ability to access lexical-semantic memory store \\
Rey's 15 word delayed recall & Language \\
Word fluency & Conceptual reasoning \\
Phrase construction & Short-term visuoperceptual recognition memory \\
Raven's 47 progressive matrices & Constructive apraxia \\
Immediate visual memory & \\
Freehand copying of drawings & \\
Copying drawings with landmarks & "Hippocampal" episodic memory \\
FCSRT & \\
FCSRT immediate free recall & \\
FCSRT immediate total recall & \\
FCSRT delayed free recall & \\
FCSRT delayed total recall & \\
FCSRT index of sensitivity of cueing & \\
\hline MMSE: Mini Mental State Examination (Folstein et al., 1975); FAB: Frontal Assessment Battery \\
(Ronga et al., 2004);; BDI: Beck Depression Inventory (Sica and Ghisi, 2007); MDB: Mental \\
Deterioration Battery (Carlesimo et al., 1996); FCSRT: Free and Cued Selective Reminding Test \\
(Frasson et al., 2011).
\end{tabular}

plied Sciences and Intelligent Systems (Rucco et al., 2020; Rombetto et al., 2014). 154 SQUIDs are placed as close as possible to the head of the subjects, the remaining ones are organized into three triplets, positioned further away from the helmet as to measure the environmental noise.

MEG data were acquired during two eyes-closed resting state segments, each $3.5 \mathrm{~min}$ long, with a minute distance between them. During the acquisition, subjects were seated inside a magnetically shielded room (AtB Biomag, Ulm, Germany) in order to reduce the external noise. Using Fastrak (Polhemus ${ }^{\circledR}$ ) we digitalized the position of four anatomical landmarks (nasion, right and left pre-auricular points and vertex of the head) and the position of four reference coils (attached to the head of the subject), in order to define the positions of the head under the helmet. The coils were activated and the position of the head was checked before each segment of registration. During the acquisition, we recorded also the cardiac activity and the eyes movements in order to remove physiological artefacts. After an anti-aliasing filter, the data were sampled at $1024 \mathrm{~Hz}$.

The MEG data were filtered in the $0.5-48 \mathrm{~Hz}$ band using a 4thorder Butterworth IIR band-pass filter, implemented offline using Matlab scripts within the Fieldtrip toolbox (Oostenveld et al., 2011). As described previously (Lardone et al., 2018), Principal Component Analysis was applied to reference SQUID signals to remove the environment noise (Sadasivan, 1996). Specifically, the filter orthogonalized the reference signals and projected the brain sensors on the basis of the noise, to clean the data (de Cheveigné and Simon, 2007). We adopted the PCA filtering implementation available within the Fieldtrip Toolbox (Oostenveld et al., 2011). Subsequently, noisy channels and bad segments of acquisition were identified and removed through visual inspection by an experienced rater. Finally, we decomposed the signal in its independent components using Independent Component analysis, identified physiological artifacts, such as eye blinking and heart activity, by visual inspection, and removed them. In particular, we found that one (sometimes two) ECG and zero (very rarely one) EOG components were present. $5 \mathrm{MCI}$ patients and $4 \mathrm{HS}$ were excluded by an expert evaluator due to excessive noise in their recordings.

\subsection{Source reconstruction}

Firstly, to reconstruct time series related to the centroids of 116 regions-of-interest (ROIs), derived from the Automated Anatomical Labeling (AAL) atlas (Tzourio-Mazoyer et al., 2002), we used the volume conduction model described in (Nolte, 2003) and the Linearly Con- strained Minimum Variance (LCMV) beamformer algorithm (Van Veen et al., 1997) (for details see (Rucco et al., 2019)), based on the native MRIs. The solutions were computed for the centroid of each ROIs, as in (Hillebrand et al., 2016). Singular value decomposition was applied to obtain a scalar value. Then, we filtered the time series in the five classical frequency bands (delta $(0.5-4.0 \mathrm{~Hz})$, theta $(4.0-8.0 \mathrm{~Hz})$, alpha $(8.0-13.0 \mathrm{~Hz})$, beta $(13.0-30.0 \mathrm{~Hz})$ and gamma $(30.0-48.0 \mathrm{~Hz}))$. Fig. 1 shows the data analysis pipeline.

\subsection{Functional connectivity measurements}

As connectivity measurements we used three amplitude-based and three phase-based metrics. Specifically, as amplitude-based metrics we used i) the classical functional connectivity based on the Pearson's correlation between brain signals ( $\mathrm{FCr}$ ); ii) Amplitude envelope correlation (AEC) (Brookes et al., 2011) which computes the amplitude envelope by means of the Hilbert transform and then determines functional connectivity between brain signals through the Pearson correlation coefficient; iii) the orthogonalized Amplitude Envelope Correlations (AECc) with signal leakage correction (Brookes et al., 2012).

As phase-based metrics we considered i) the Phase Lag Index (PLI) which estimates the asymmetry of the distribution of the phase differences between the brain signals (Stam et al., 2007); ii) the weighted Phase Lag Index (wPLI) which weights the PLI by the magnitude of the imaginary component of the cross-spectrum (Vinck et al., 2011); iii) the Phase Linearity Measurement (PLM) which measures the synchronization between brain regions by monitoring their phase differences in time (Baselice et al., 2019). Please note that FCr and AEC do not correct for volume conduction, while AECc, PLI, wPLI and PLM do. The phase linearity measurement is a phase-based synchronization measure ranging from 0 (no synchronization) to 1 (synchronization), insensitive to volume conduction. It captures similar information as metrics such as the PLV or the PLI, but requires less data to give a reliable estimate (Baselice et al., 2019). Unlike these metrics, the PLM is based on the spectrum of the interferometric signal between any pair of brain regions. In conclusion, for each subject and each metric, we obtained two testretest connectomes.

\subsection{Towards clinical connectome fingerprinting}

The methodology for clinical connectome fingerprinting is inspired by recent work on maximization of connectivity fingerprints in human functional connectomes in health (Amico and Goñi, 2018) and disease 


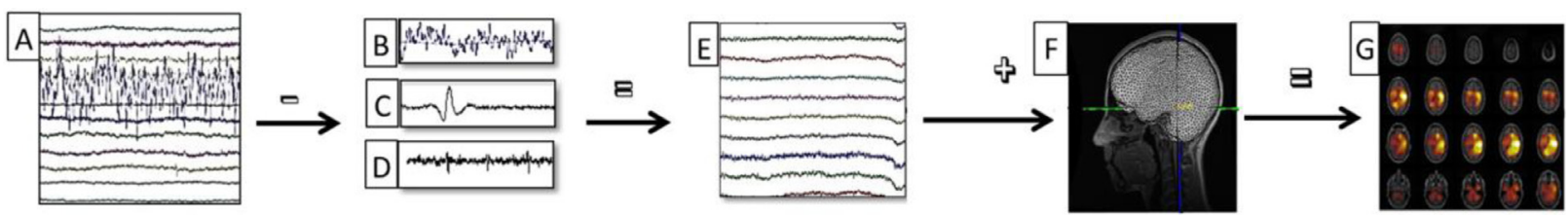

Fig. 1. Data analysis pipeline. (A) Raw MEG signals recorded by 154 sensors (a subset displayed here). (B-C-D) Respectively noisy channel, cardiac artifact, blinking artifact, removed during preprocessing phase. (E) MEG signals after noise cleaning and artifact removal. (F) Co-registration between MEG signals and MRI. (G) Source reconstruction (beamforming).

A. Clinical Connectome Fingerprinting
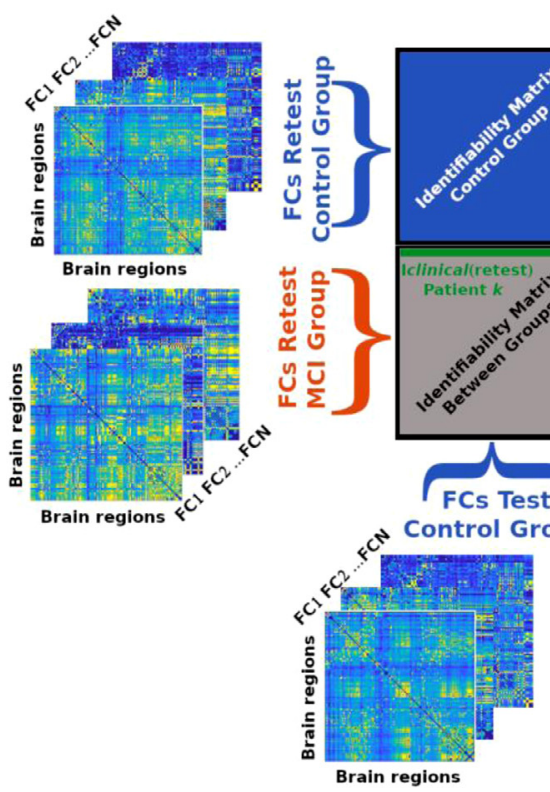

\section{B. Association with clinical scores}
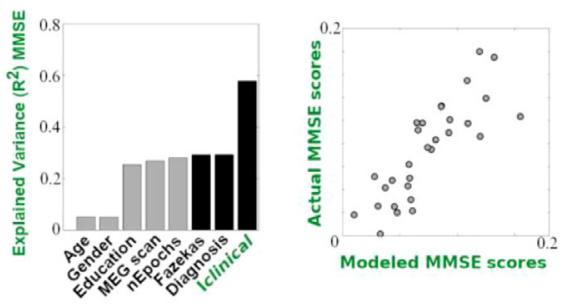

\section{Prediction of clinical scores from Clinical Fingerprinting}

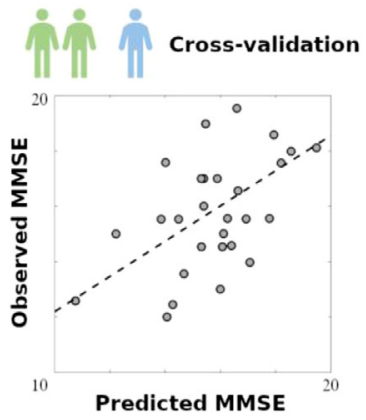

Fig. 2. Clinical connectome fingerprinting scheme. A) The Identifiability matrix (Amico and Goñi, 2018) is computed for each group, using the test-retest individual

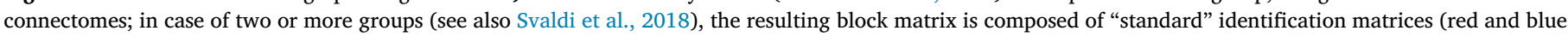

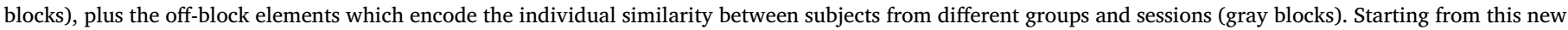

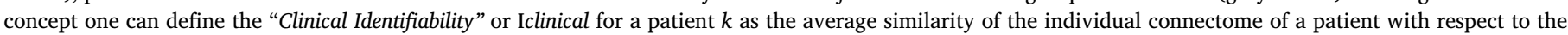

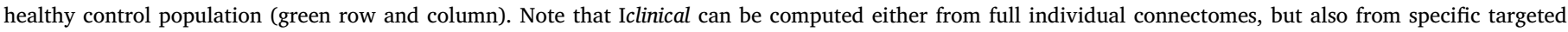

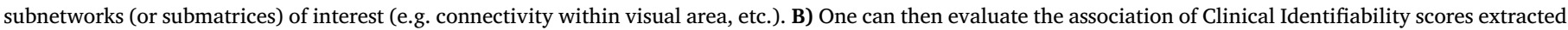

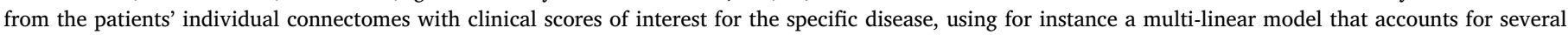

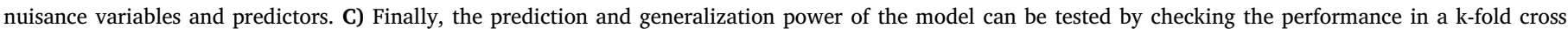
validation fashion.

(Svaldi et al., 2018). Briefly, it starts from defining the mathematical object known as "Identifiability" or "Identification" matrix (Amico and Goñi, 2018) see also Fig. 2A). In this context, the similarity is defined as the Pearson's correlation between the connectomes at hand. The identifiability matrix has subjects as rows and columns, and encodes the information about the self similarity (Iself, main diagonal elements) of each subject with herself/himself, across the test/retest sessions, and the similarity of each subject with the others (or Iothers, off diagonal elements) (Amico and Goñi, 2018). The difference between the average Iself and the average Iothers (denominated "Differential Identifiability" or "Differential Identification" - Idiff (Amico and Goñi, 2018)) provides a robust score of the fingerprinting level of a specific dataset (Amico and Goñi, 2018).

This framework can easily be extended in scenarios where multiple clinical groups are present [39] see also Fig. 2A). In this case, the Identifiability matrix becomes a block matrix, where the number of blocks equals the number of groups (i.e. two in the case of this work, Fig. 2A). The within-group blocks (blue and red blocks in Fig. 2A) represent the Identifiability matrix within a specific clinical group (i.e. MCI or healthy controls). The between blocks (groups) elements (i.e. the two gray blocks in Fig. 2A) encode the similarity (or distance) between the test-retest connectomes of subjects belonging to different groups. In particular, the top right block contains the similarities between the connectomes from patients during the test session with the connectomes of the controls during the retest session, while the bottom-left block contains the opposite case (i.e. the similarities between the connectomes of the patients during the re-test session with the connectomes of the controls during the test session). Let $C$ be the set of the healthy volunteers. Similarly, let $G$ define the patient group. Also, let I be the Identifiability matrix depicted in Fig. 2A. Hence, we can define Iclinical(test) and I clinical (retest), for a specific patient $k$, as:

Iclinical $_{k}($ test $)=\frac{1}{N_{C}} \sum_{i} I_{i k} ;$ Iclinical $_{k}($ retest $)=\frac{1}{N_{C}} \sum_{i} I_{k i}, \forall i \in C$

To summarize, Iclinical(test), for each patient $k$, represents the average similarity of the connectome of that patient in the test session with the connectomes of every control in the retest session, and Iclinical(retest) represents the average similarity of the connectome of a patient in the retest session with the connectomes of every control in the test session. Taking advantage of the new piece of information provided by the between groups blocks, we define the "Clinical Identification" or "Clinical 
Identifiability" (Iclinical), for patient $k$, as:

Iclinical $_{k}=\frac{1}{2}\left(\right.$ Iclinical $_{k}($ test $)+\operatorname{Iclinical}_{k}($ retest $\left.)\right), \forall k \in G$

Essentially, for each patient $k$, Iclinical provides the (average) score of how similar is their connectome with respect to the control subjects in the population, across the test-retest sessions.

\subsection{From clinical connectome fingerprinting to prediction of clinical scores}

Edgewise Intraclass correlation. For each group (HS and MCI) we quantified the edgewise reliability of individual connectomes using intraclass correlation (ICC, (Koch, 2014), similarly to previous work (Amico and Goñi, 2018). ICC is a widely used measure in statistics, normally to assess the percent of agreement between units of different groups. It describes how strongly units in the same group resemble each other. The stronger the agreement, the higher its ICC value. We used ICC to quantify to which extent the connectivity value of each edge (functional connectivity value between two brain regions) could separate within and between subjects. In other words, the higher the ICC of an edge, the more stable that edge's connectivity across the test-retest session and, in turn, the higher the edge's "fingerprint" (i.e. that particular edge is relevant to identify individual connectomes). We then go on and test if the edge's identifiability diminishes in patients, and if such reduction is predictive of individual clinical disability.

Multilinear model specification. To test for the hypothesis that clinical connectome identification is associated with clinical scores, we performed a multi-linear regression analysis to predict the MMSE scores based on Iclinical and two other predictors. Specifically, a categorical variable encoding diagnosis (amnestic MCI and multi domain MCI), and the Fazekas index which quantifies the amount of white matter T2 hyperintense lesions (i.e. vascular burden). Five nuisance variables were also included to account for any potential effects of age, sex, education, different day of MEG scans, and different number of epochs.

Edge selection and prediction of clinical scores. The Clinical Identification scores defined earlier (Fig. 2A) can be computed from full individual connectomes, but also from a subset of the individual connectomes (i.e. by computing Patient/Controls similarity only on a subset of edges). Furthermore, if the reduction of identifiability is related to the pathological processes, we expect that the individual level of fingerprinting would be predictive of the individual clinical impairment, and maximally so when based on the subset of most reliable edges. Therefore, we tested the specificity of the prediction, as well as the generalization capacity of our model, by using a k-fold cross validation $(k=5)$ approach. The approach detailed below has some similarities with the Connectome Predictive Modeling methodology (CPM, (Shen et al., 2017)), with two major key differences.

Firstly, we selected the connectome edges to be included in the fingerprinting based on the edgewise ICC value computed on the control group. That is, edges were ranked in descending order according to the ICC value, and only a subset was included in the fingerprint analysis (similarly to (Amico and Goñi, 2018)). Note that, in order to improve the signal-to-noise ratio and avoid source reconstruction artefacts, edges entirely within the cerebellum were not included in the analysis. The k-fold validation was then performed iteratively by adding 100 edges at the time, starting from the most reliable edges (as measured by ICC), and ending with the least robust ones, until eventually taking into consideration the full individual connectomes. Secondly, as aforementioned, at each iteration the individual Iclinical scores, based on the iteration-specific subset of edges, were used to predict the individual MMSE scores. As said, depending on the number of edges included, the Iclinical represents the similarity with (or distance from) the control group relative to the specific connectome subcircuit spanned by the included edges. Finally, the prediction scores between the multi-linear (ML) model with Iclinical and MMSE clinical scores were evaluated for each of the five frequency bands studied.
Null models for prediction. In order to make sure that the edge selection based on the ICC scores is clinically meaningful, we implement two different null models. In the first one (named Null-Edges), we built a distribution of the prediction scores based on randomly selected edges. Hence, at each step of the k-fold model we shuffled the ICC mask 1000 times, and recomputed the prediction scores between the Iclinical multilinear model and MMSE. In other words, we build a "null distribution" of prediction rates, entirely based on a randomized edge selection, however starting from the empirical functional connectomes. In the second null model (named Null-MMSE), we are accounting for the sample size effect when performing k-fold validation. To this end, we performed permutation testing on the MMSE scores, by randomly shuffling them 1000 times, and by computing the corresponding confidence intervals related to the obtained null distribution. Prediction scores outside of the 95 percentile of the Null-MMSE distribution were considered as significant predictions for our model. We chose to use the k-fold validation since it has been shown - both theoretically and empirically - to improve prediction estimates in small neuroimaging datasets, as compared to leaveone-out cross validation (LOOCV) (Varoquaux, 2018; Varoquaux et al., 2017). However, the LOOCV validation was also carried out, and in this dataset LOOCV showed similar results, as shown in the SI Figure S5.

\section{Results}

We tested the Clinical Connectome Fingerprinting (CCF, Fig. 2) framework on a resting-state MEG dataset acquired from an elderly cohort of 69 subjects, 34 healthy controls and 35 affected by amnestic Mild Cognitive Impairment. The test-retest sessions were acquired during the same day, with a $\sim 1$-minute break from each other. From the initial population of 69 subjects we excluded those who: 1) were affected by noise or 2) did not have two test-retest sessions. This left us with 30 subjects per group, a total of 60 subjects.

Clinical connectome fingerprinting builds upon recent work on maximization of connectivity fingerprints in human functional connectomes (FCs) in health (Amico and Goñi, 2018) and disease (Svaldi et al., 2018). As explained, the first step of CCF is to construct the "Identifiability" matrix (Amico and Goñi, 2018), see also Fig. 2A and Methods), for the "combined" clinical and healthy population. In this case, the Identifiability matrix becomes a block matrix, where the number of blocks equals the number of groups (i.e. two in the case of this work, Fig. 2A and Methods). On one hand, each block represents the identification within a specific clinical group (blue and red blocks in Fig. 2A). On the other hand, the between blocks (groups) elements (i.e. in the case of this paper, the two gray blocks in Fig. 2A) encode the similarity (or distance) between connectomes of subjects belonging to different groups (i.e., Iclinical, see Methods for details), for both the test and retest session. Essentially, for each patient, Iclinical provides the (average) score of how similar their connectome is with respect to the control subjects in the population, as well as across test-retest sessions (Fig. 2A). The major hypothesis behind this work is that the Iclinical scores can be representative of the connectome degeneration associated with the disease, and therefore associated with the behavioral/clinical scores at hand (Fig. 2B).

In order to test for that, the first step was to select the best metric for fingerprinting the MEG functional connectomes. We therefore evaluated the fingerprinting capacity of six popular network metrics for MEG connectomics. Three of these were amplitude-based (Amplitude based correlation (AEC, (Brookes et al., 2011)); AEC corrected for spatial leakage (AECc, (Brookes et al., 2012)); Pearson correlation), and three were phase-based measurements (Phase Lag Index (PLI, (Stam et al., 2007)); weighted PLI (wPLI, (Vinck et al., 2011)); Phase Linearity Measurement (PLM, (Baselice et al., 2019)), Fig. 3 and S1). In this regard, differential Identifiability (Idiff, (Amico and Goñi, 2018), see also Methods) provides a good score to test the robustness and reliability of each connectivity measurement across sessions.

Fig. 3 shows the results of the fingerprinting test: the PLM-based Iself scores appear to be the most reliable with respect to the other met- 
A

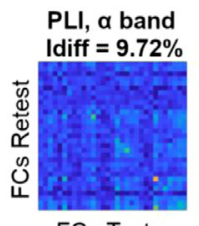

FCs Test

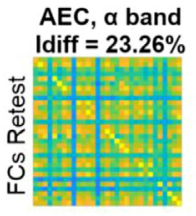

FCs Test

\section{B}

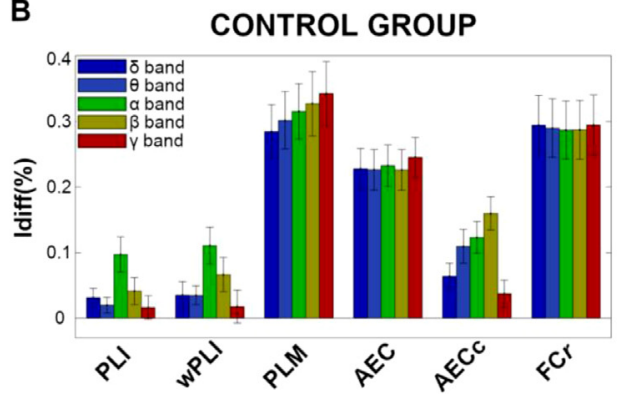

CONTROL GROUP

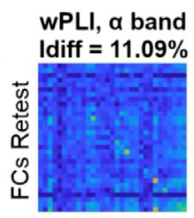

FCs Test

AECc, $\alpha$ band

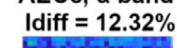

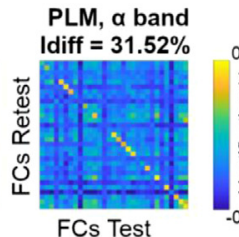

FCs Test

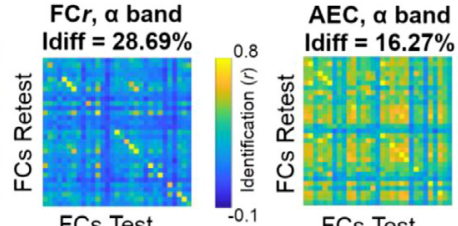

FCs Test

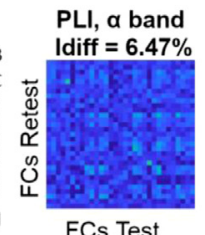

\section{GROUP}

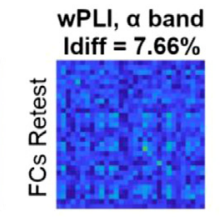

FCs Test

AECc, $\alpha$ band
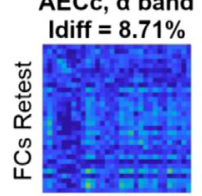

FCs Test
PLM, a band
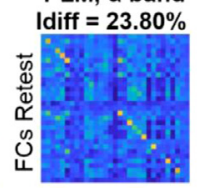

FCs Test

$\mathrm{FC} r$, a band

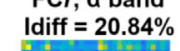

MCI GROUP

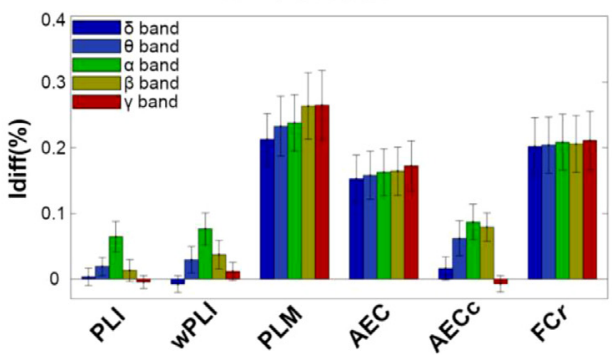

Fig. 3. Data-driven selection of the most reliable connectivity metric for clinical connectome fingerprinting. A) Identifiability matrices for the HS and MCI group, for each of the six connectivity metrics tested: Phase Lag Index (PLI), weighted PLI (wPLI), Phase Linearity Measurement (PLM), Amplitude Envelope Correlation (AEC), AEC corrected for spatial leakage (AECc), Pearson's correlation (FCr). Here only the alpha band is shown (the other bands are reported in Fig. S1). The differential identification score (Amico and Goñi, 2018) is used to select the best metric for clinical connectome fingerprinting in this MEG dataset. B) The Idiff scores across bands and connectivity metrics are summarized for the two groups; note how PLM outperforms all the other methods in all the frequency bands evaluated. We hence selected PLM connectomes for the fingerprinting analyses that follow. rics (paired $t$-test $p<0.05$ in both Control and MCI groups, Bonferroni corrected across frequency bands and metrics tested, see also Fig. 3B.; please see Fig. S2 for a comparison between PLM and PLI). For an analysis of the identification rates, that yields similar results, please refer to Fig. S3. Interestingly, there is also a consistent drop in Idiff scores when comparing the MCI group with the HS (Fig. 3A). We therefore selected the PLM as the most robust method for the connectivity fingerprinting on this MEG dataset.

We then explored the local specificity of MEG fingerprinting in PLMbased individual connectomes, by using intraclass correlation (ICC) on the functional connectome edges (see Amico and Goñi, 2018 or Methods for details), across frequency bands. Note that, in order to ease the visualization of the results, hereafter we will only show results from the three frequency bands, namely theta, alpha, and beta. The results for the other two (delta and gamma) are reported in SI (Fig. S4). The analysis on the spatial specificity of the connectome fingerprinting is depicted in Fig. 4. Note the consistent drop in ICC values when comparing the ICC edgewise patterns of the HS group to the MCI group (Fig. 4).

The results reported in Fig. 4 made us speculate that a decrease in fingerprinting might be also associated with cognitive decline in the MCI population. Specifically, we sought to test the hypothesis that the individual patient's connectome similarity/distance scores from the healthy connectomes (i.e. Iclinical, see Methods), particularly when restricted between subsets of highly reliable edges, could be used as biomarkers of cognitive decline.

We therefore tested the clinical connectome fingerprinting framework on the individual PLM matrices computed for the two groups, across all frequency bands. Briefly, we tried to predict MMSE scores from the Iclinical similarity scores obtained from comparing connectivity subsets of most reliable edges (in an iteratively increasing fashion, from 50 to the entire functional connectomes, adding 50 edges at each iteration, analogously to (Amico and Goñi, 2018)) between each MCI patient and the HS population. These Iclinical scores were added into an additive multi-linear model to account for the possible confounds and nuisances in the dataset (Fig. 5B). To test for the generalization of the prediction, $\mathrm{k}$-fold cross validation $(k=5)$ was performed at each iteration, and the prediction score (Spearman's $\rho$ between predicted vs. observed MMSE, Fig. 5) was tested against the prediction score derived from two different null models: one obtained by randomly permuting the edge subset at each iteration 1000 times; the other by randomly permuting the MMSE scores 1000 times, also called permutation testing in the machine learning community (see also Methods for details). The 95\% upper limit of the confidence interval obtained from permuting the MMSE labels is represented by the dashed black lines in Fig. 5A. We found that the Iclinical-based linear model significantly predicts the MMSE in the alpha band, with a peak in prediction when using the top 300 most reliable edges (Fig. 5A). Iclinical scores in the training set are significantly associated with the MMSE scores $\left(p=0.0005, \mathrm{R}^{2} \simeq 0.6\right.$, Fig. 5B), with positive beta coefficients. That is, the higher the Iclinical score of the MCI patient (i.e. the more similar to the HS cohort their selected subnetwork is), the higher their MMSE score. Interestingly, despite the use of a simple linear model, both the k-fold validation and the LOOCV results show good generalization and prediction capacity of the MMSE from connectome features, both at peak (300 edges, Spearman's $\rho=0.55, p<0.05$ Bonferroni corrected across bands), or when including more edges in the selected subnetwork (e.g. 700 edges, $\rho=0.51, p<$ 0.05 Bonferroni corrected, Fig. 5C, see also S5, S6). The brain regions involved in the maximal prediction spread over the entire brain network: from frontolateral cortices, to occipital, to even cerebellar connections (Fig. 5C). Notably, prediction at alpha is significantly different from the null model based on edge permutation, as well as from the null model obtained by shuffling the MMSE scores, since the maximal prediction found is well above the confidence interval (despite the relatively small sample size, Fig. 5A).

\section{Discussion}

In the current manuscript, we aimed to test the hypothesis that the regulation of the pattern of large-scale brain interactions is weakened in amnestic mild cognitive impairment (aMCI). Hence, we reasoned that, if the features of the functional connectome are less efficiently regulated in $\mathrm{MCI}$, then the connectomes might be less easily recognizable or "identifi- 
CONTROL GROUP
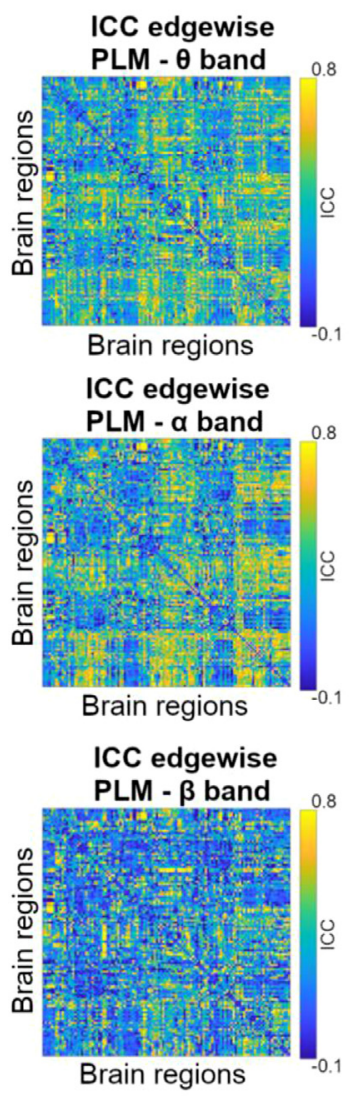
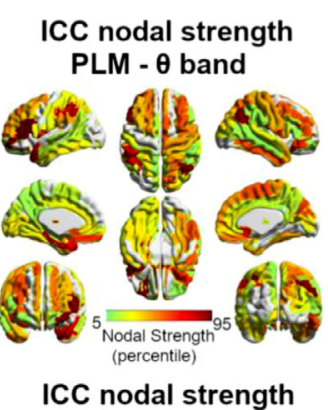

PLM - $\alpha$ band

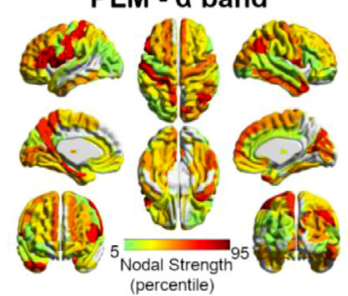

ICC nodal strength PLM - $\beta$ band

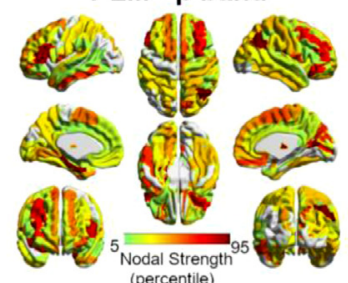

(percentile)
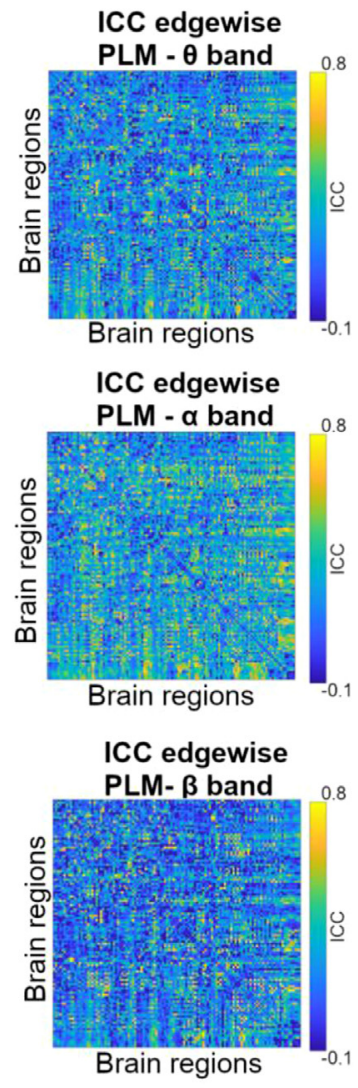

MCI GROUP

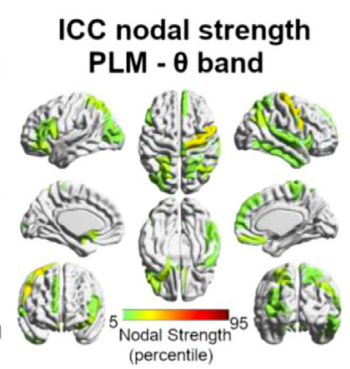

ICC nodal strength

PLM - $\alpha$ band

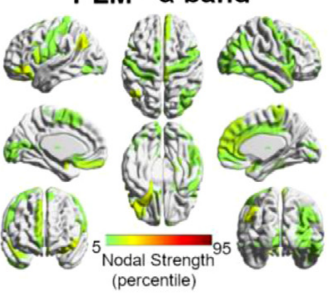

ICC nodal strength

PLM - $\beta$ band

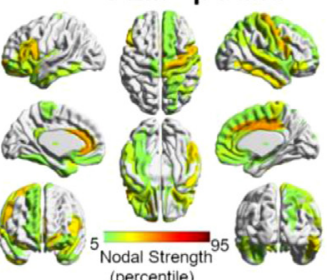

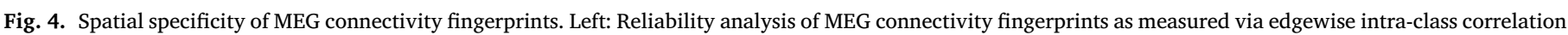

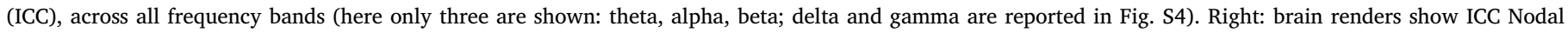

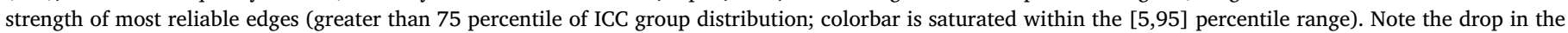
ICC distribution values when comparing the healthy control group to the MCI one.

able". We therefore defined a novel framework, namely Clinical Connectome Fingerprinting (Fig. 2), to extract individual features from diseased connectomes (or relevant subnetworks), and use them as biomarkers for prediction of mild cognitive impairment in a MEG dataset of an aMCI population.

Firstly, we compared the identification performance of a number of commonly used MEG connectivity metrics in both the healthy and aMCI cohort. Specifically, the metrics chosen were the phase lag index (PLI) (Stam et al., 2007), the weighted phase lag index (wPLI), the amplitude envelope correlation (AEC) (Brookes et al., 2011), the orthogonalized amplitude envelope correlation (AECc) (Brookes et al., 2012), the Pearson correlation directly computed on the time series (FCr), and the phase linearity measurement (PLM) (Baselice et al., 2019). Of these metrics, the FCr, AEC and AECc are amplitude-based, while the PLI, wPLI and PLM are phase-based. Furthermore, FCr and AEC do not correct for volume conduction, while AECc, PLI, wPLI and PLM do (although AECc uses a different approach to do so - i.e. orthogonalization (Brookes et al., 2012)). We used source-reconstructed, resting-state MEG signals, and compared the fingerprinting capacity of the aforementioned metrics (Fig. 3). The PLM performs significantly better than the other metrics (paired $t$-test $p<0.05$ in both Control and MCI groups, Bonferroni corrected across frequency bands and metrics tested, Fig. 3B; see also S3 for an analysis of the identification rates, which show similar results). As previously known, amplitude-based metrics tend to outperform phase-metrics in terms of noise-resiliency (Colclough et al., 2016), and metrics that do not correct for volume conduction outperform those who do in terms of identifiability (Demuru et al., 2017), perhaps because they include information that is subject-specific though unrelated to genuine brain activity. However, PLM seems to be an exception to these trends, being a purely phase-based metric that corrects for volume conduction, possibly because PLM is more robust to noise and can give reliable connectomes with shorter MEG acquisitions (Baselice et al., 2019). Notably, among the connectivity metrics correcting for spatial leakage, PLM is also the one that achieves the best identification rates, in both the control and MCI groups (Fig. S3). These features might be more related to genuine neural activity and less influenced by the geometry of the head (Colclough et al., 2016). Importantly, the analysis of fingerprints based on MEG data allows to exploit the temporal richness of neuronal interactions (as measured through phase synchronization), enriching the insights provided by the broader fMRI-based fingerprinting literature.

Once we spotted the best connectivity metric, inspired by recent work on connectome fingerprinting (Amico and Goñi, 2018; Svaldi et al., 2018), we tested if subject identification would be harder to perform on the MCI group as compared to controls. As shown (Fig. 4, Fig. S1), the identifiability of the patients drops drastically as compared to controls. Previous evidence showed that the large-scale activity in the healthy brain is fine-tuned to achieve both efficient communication and functional reconfiguration, which underpins complex, adaptable behavioural responses (Deco et al., 2009; Sorrentino et al., 2019). Therefore, this finding might be framed within the dysregulation of large-scale activity due to the pathological processes. Less regulated activity might imply less stable or reliable activity, which might induce lower similarity between test-retest connectomes of MCI subjects. In turn, this might imply the reduced edgewise identifiability that we observed (Fig. 4, Fig. S1). 
A

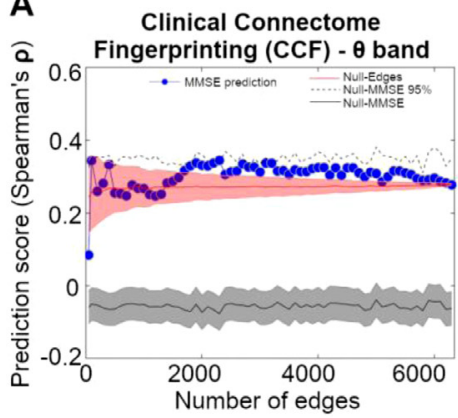

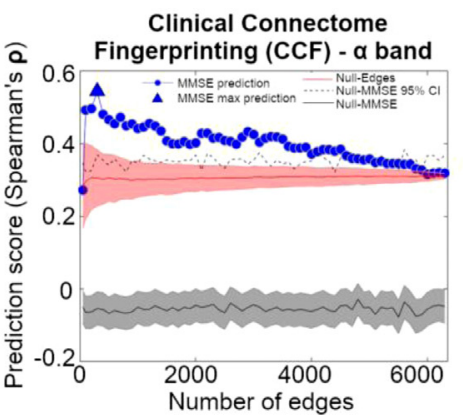

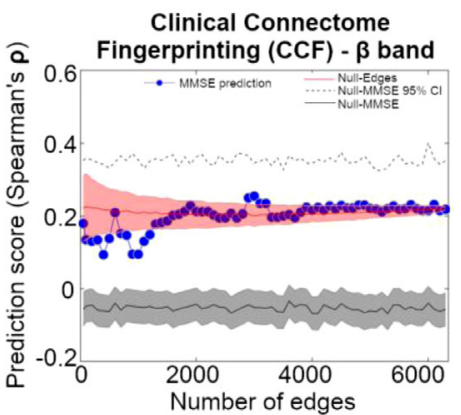

B

Multilinear model at peak $\mathbf{\Delta}$ (300 edges) MMSE - $\alpha$ band
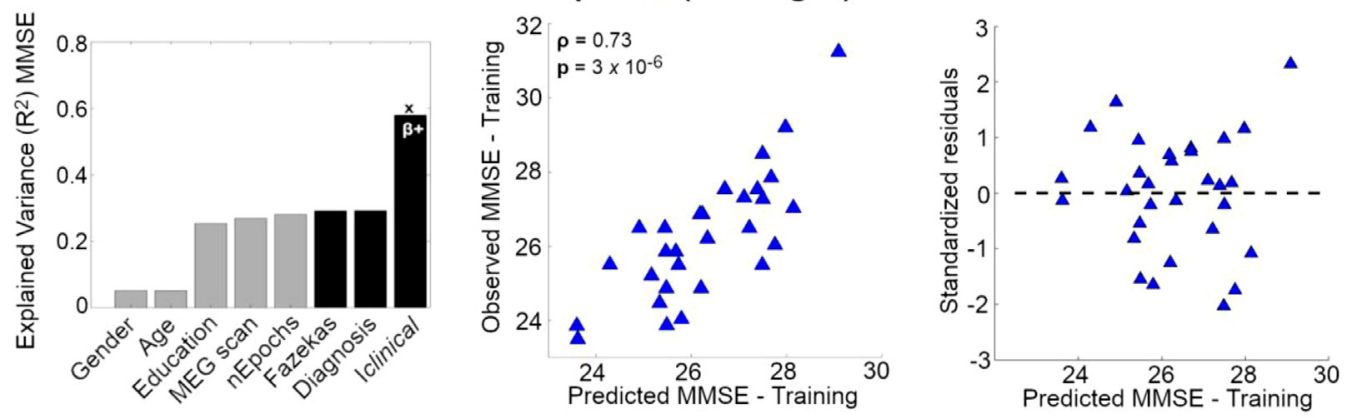

\section{C}

Prediction at peak $\Delta$ (300 edges) MMSE - $\alpha$ band

Prediction at 700 edges MMSE - $\alpha$ band
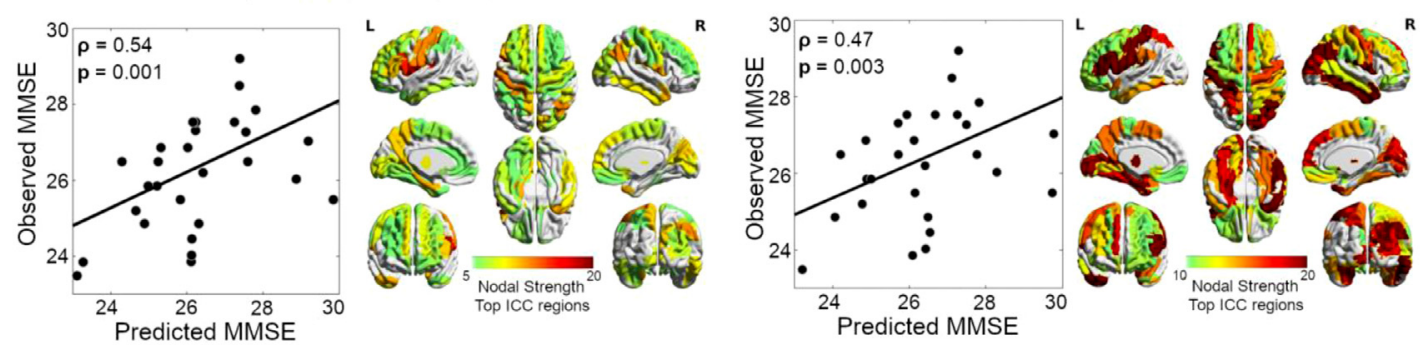

Fig. 5. Clinical Connectome Fingerprinting for Mini Mental State Examination (MMSE) prediction. A) Feature selection based on ICC. At each frequency band, subset of edges are added iteratively (from 50, 100 to whole-brain, in step of 100) based on their ICC values, from most to least reliable (x-axis), and prediction performance ( $\mathrm{k}$-fold $(k=5)$ cross validation, see Methods) of the multi-Linear model based on Clinical Identifiability (Iclinical) is evaluated (y-axis), and compared against two null models: one (Null-Edges, red line), obtained by randomly choosing the subset edges 1000 times at each step (shaded red line denotes its standard deviation); the second (Null-MMSE, black line), obtained by randomly permuting the MMSE scores 1000 times at each step (shaded gray line indicates standard error; dashed black line denotes 95\% confidence interval for Null-MMSE). B) Multi-linear model at peak. The performance of the model training set is shown for the peak prediction (300 edges, alpha band). Left: The additive linear model consists of five nuisance variables (Gender, Age, Education, Meg scan, number of Epochs), and three predictors (Fazekas index, Diagnosis, Iclinical, see also Methods). Significant predictors are indicated by the $\mathbf{x}$ ( $p<0.05$, Bonferroni corrected across frequency bands); $\beta+$ indicates that the beta coefficients for Iclinical are positive (i.e. the higher Iclinical, the higher the correspondent MMSE score). Center: Scatter plot of the Observed MMSE scores versus the MMSE scores predicted by the multi-linear regression model. Right: Scatter plot of the standardized residuals versus the predicted MMSE scores for the multi-linear model. Note how the residuals are symmetrically distributed, tending to cluster around 0 , and within 2.5 standard deviations of zero. C) Nodal degree of most predictive brain regions. Figure shows prediction scatter plot for the k-fold test set, at peak (300 edges, alpha band) and at another local maximum (700 edges, alpha bands). The correspondent brain renders represent the nodal degree associated with the selected edge mask at 300 and 700 edges, respectively.

The ICC nodal strength complements the picture of MCI altered connectivity in terms of regional activity, since not all brain regions contribute equally to subject identification (Fig. 4, Fig. S4). Furthermore, we wish to stress that strongest connectivity, as depicted by the ICC results in Fig. 4, does not necessarily relate to strongest reliability (Fig S2). For instance the occipital regions in alpha, which are among the strongest in terms of PLM connectivity (Fig. S2A), are not the strongest in terms of the ICC. Therefore, connectivity and fingerprints provide complementary, but distinct information. Interestingly, the pattern of regions that contribute the most to identifiability varies according to the frequency band. Our results point toward the presence of relevant information in the alpha band, as well as in the occipito-temporal and occipito-parietal regions. Previous evidence showed that the alpha band carries relevant information to the conversion of MCI, both in changes in power (Moretti et al., 2007) and in synchronization patterns (López et al., 2014), and that hyperactivations in the dorsal and ventral pathways (i.e. occipito-temporo-parietal areas) characterize MCI subjects (Maestú et al., 2011). It is noteworthy that some of these regions overlap consistently with previous findings in fMRI fingerprinting (Amico and Goñi, 2018). However, some other areas, such as associative visual cortices (Fig. 4), which exhibit high identification power in this dataset, are not so important for fMRI fingerprinting (Finn et al., 2015). This led us to speculate that connectivity fingerprinting might also depend on the modality used to measure it (e.g. MEG as opposed to fMRI), which might also reflect the specific time scale of neuronal interactions/synchronies. Future studies should deepen the investigation on the relationships between fingerprinting and neuroimaging modalities, as well as on exploring other conditions, such as eyes-open. 
More importantly, the fingerprinting regions highlighted in Fig. 4 encompass associative areas: that is, they integrate multiple information sources to plan coherent, complex behavioural responses (Corbetta and Shulman, 2002; de Pasquale et al., 2012). We then hypothesized that impaired regulation of the interaction of such brain regions should lead to poorer cognitive performance, as well as poorer identification. If this is the case, the harder it is to identify a subject, the worse its cognitive performance should be. It is reasonable to assume that not all edges are equally important for brain fingerprinting, and that not every edge is equally affected by the pathological processes occurring in MCI. However, there might exist an overlap between these two subsets. This was indeed our working hypothesis for clinical identification.

In MCI and Alzheimer's disease, the Mini Mental State Examination (MMSE) is one of the most widely used bedside assessments of cognitive function (Folstein et al., 1975). We use clinical connectome fingerprinting to show that there is a strong correlation between the MMSE score and clinical identification in the alpha band (Fig. 5A), even when taking into account confounders such as age, education, time of the acquisition, length of the scan, and subject-specific vascular burden (Fig. 5B). Furthermore, the linear model based on clinical fingerprinting scores significantly predicts the MMSE scores on the k-fold validation (Fig. 5A, Fig. 5C). Notably, the optimal prediction is achieved when considering the first 300 most reliable edges (shown in Fig. 5). As one can observe, after including all the other covariates, the $\mathrm{R}^{2}$ of the model drastically increases when Clinical identifiability is taken into account (Fig. 5B). This shows that Clinical Fingerprinting might capture some processes related to cognitive performance (Noble et al., 2019) in MCI. Moreover, our results on Clinical Fingerprinting are obtained from a phase-based connectivity metric (PLM), which might represent specific mechanisms of communication, i.e. phase synchronization (Engel et al., 2013). Furthermore, the alpha band, where the best prediction occurs (Fig. 5, Fig. S3), had been previously shown to be altered in MCI (Jacini et al., 2018; Engels et al., 2017). Furthermore, note that the MMSE prediction failed when using standard edgewise connectivity values, as opposed to Iclinical scores (see Fig. S7).

The result that the most reliable edges (high ICC (Noble et al., 2019)) are also predictive of an MCI-related cognitive outcome (MMSE, Fig. 5) is an interesting one. Keep in mind that, with our data-driven methodology purely based on ICC, one cannot have control over the kind of features that are being selected for the subsequent prediction of clinical outcomes (Hartoyo et al., 2019). In fact, one can see that adding a few edges - despite them being the most reliable ones, as edges are being added sequentially according to their ICC - does not guarantee the best prediction. Notably however, once a sufficient number of edges has been added, one reaches the best prediction (Fig. 5A). Presumably, this prediction includes a set of edges that underpin the mild cognitive impairment tested with MMSE. Similarly, adding more edges does not improve the prediction further, but rather makes it slowly decline (Fig. 5A). Hence, adding more (less identifiable) edges means to be adding irrelevant information for the prediction of the behavioral outcome under study. Again, our results in this MCI cohort show that the most reliable edges are also the most predictive ones, clinically. If these edges were not specifically related to the cognitive output, then a randomly selected subset of edges should perform similarly in terms of predictive power on the MMSE score. This does not seem to be the case, as the null model results show (Fig. 5A, red shaded line). In fact, when considering random edge selections, the quality of the prediction drops drastically, showing that the ICC selects those edges that are informative with respect to cognitive performance, as tested through MMSE (Fig. 5A, red shaded line). The reduction in identifiability between connectomes in both the MCI and control groups does not appear to be driven by head movement, as measured by distance in the squid positions between the beginning of the first and second acquisition (double-sided $t$-test between groups non significant). Furthermore, there is no correlation between Iself and the aforementioned motion scores (see also Fig. S8). Hence, it seems that differences in movements are unlikely to justify the differences between the two groups. However, it is important to note that continuous head tracking was not available in this study, and this should be considered as a major limitation in assessing motion differences between groups. Finally, the fingerprinting results reported here do not appear to be driven by simple differences in power between groups or changes to the slope of $1 / \mathrm{f}$ aperiodic neural activity (Fig. S9, Kolmogorov-Smirnov test between groups non significant).

The findings of this study make it essential to lay out several methodological considerations. The first one relates to the reliability/validity "dichotomy" (Noble et al., 2019). Here we show that edges that are most reliable possess a strong clinical validity for cognitive impairment prediction (Fig. 4). However, the reader should keep in mind that robust edges in MEG data can be associated with several factors, not all of them necessarily neuronal-related: motion artifacts, gray matter atrophy, individual source reconstruction parameters, epochs length, and so on. Despite our efforts in controlling for all these (as detailed in the Methods and shown in Fig. 5B), further studies should dig into the fingerprinting "causes" and properties of MEG data. The same applies to the clinical validity part of our findings: the link found between reliability/validity might be dataset and/or disease dependent, and should be explored in different populations and clinical conditions. Also, here we use a data-driven method to select the best edge features for clinical prediction, as they turn out to be significantly better than random selected features (Fig. 5A). Nevertheless, we encourage further work to explore edge selection based on a-priori hypothesis for the disease at hand, which might outperform the proposed data-driven feature selection for clinical connectome fingerprinting. Future studies should also explore the effect of denoising techniques to maximize fingerprints from brain data onto the Iclinical scores (11).

Another important caveat of this study is that, in MEG sourcereconstructed data, the signal-to-noise ratio is heavily dependent on the distance between the source and the sensor, and hence is not homogeneous for all the sources. However, recent evidence showed that signals reconstructed from the basal ganglia contain reliable information about brain activity (Müller et al., 2019; Pizzo et al., 2019) as well as those from the cerebellum (Andersen et al., 2020). On the one hand, MEG signals derived from the cerebellum are $30-60 \%$ weaker as compared to the cortical surface (Samuelsson et al., 2020). On the other hand, the cerebellum is an important structure in both motor and cognitive processes, and hence excluding it all along is likely discarding some information (Ito, 2006). In this work we tried to account for this by excluding entirely within-cerebellar links from ICC edge selection, while keeping edges that are incident but not contained within the cerebellum (see Methods for details). However, we observed that our predictions are maintained when including within-cerebellar links, while they drastically drop when excluding the cerebellum altogether from the individual connectomes (MMSE maximal prediction $\rho=0.3$, Fig. S10). One should keep in mind that our predictions involve a clinical outcome. The fact that cortical-cerebellar links are needed to improve MMSE prediction, after controlling for all nuisance factors and after benchmarking it against an appropriate null-model for edge selection, implies that they contain relevant information associated with the individual cognitive state that should not be discarded. We encourage further research to avoid the underestimation of cerebellar connectivity in MCI and AD, and explore further the role of these cortico-cerebellar pathways. In this work, we have opted to use resting-state data to test our clinical fingerprinting framework. Future work should explore Iclinical scores in task-based paradigms, which could help in maximizing detectability of the "fingerprints of cognitive decline" (Finn and Bandettini, 2021). Finally, whilst we have used a $k=5$ cross validation approach to alleviate potential over-fitting issues, as recommended in previous works (Varoquaux, 2018), we recommend future studies to explore the clinical fingerprinting framework on larger datasets (i.e. more than 30 participants in each group), whenever possible. Future work should also explore the proposed CCF approach in combination with other neuroimaging/behavioral data, for prediction purposes (Engemann et al., 2020) 
In conclusion, we have defined Clinical Connectome Fingerprinting, a novel approach to extract individual connectivity features from diseased functional connectomes. We applied this framework for clinical identification of MEG connectomes extracted from an elderly population of subjects with mild cognitive impairment (i.e., amnestic MCI). We showed that the most identifiable edges are also the most predictive of individual cognitive impairment, as measured by the Mini-Mental State Examination score. We hope that future studies will exploit further the potential of Clinical Connectome Fingerprinting as a preclinical diagnostic tool, as well as a way to empirically link, in a data-driven fashion, specific sub-networks to given cognitive functions or brain states.

\section{Data and code availability statement}

The data that support the findings of this study are available from the corresponding author upon reasonable request. The data are not publicly available due to the clinical nature of the cohort under study. The code (in MATLAB) used for this analysis will be available upon acceptance on EA EPFL webpage.

\section{Credit statement}

PS and RR collected and acquired the dataset, processed the data and conceptualized the study; EA conceptualized the study, designed the framework and performed the connectivity analyses; all authors interpreted the results and wrote the manuscript.

\section{Fundings}

EA acknowledges financial support from the SNSF Ambizione project "Fingerprinting the brain: network science to extract features of cognition, behavior and dysfunction" (grant number PZ00P2_185716).

\section{Declaration of competing interest}

The authors declare no competing financial interests.

\section{Supplementary materials}

Supplementary material associated with this article can be found, in the online version, at doi:10.1016/j.neuroimage.2021.118253.

\section{References}

Albert, M.S., DeKosky, S.T., Dickson, D., et al., 2011. The diagnosis of mild cognitive impairment due to Alzheimer's disease: recommendations from the National Institute on Aging-Alzheimer's Association workgroups on diagnostic guidelines for Alzheimer's disease. Alzheimers Dement. 7, 270-279. doi:10.1016/j.jalz.2011.03.008.

Amico, E., Goñi, J., 2018. The quest for identifiability in human functional connectomes. Sci. Rep. 8, 8254. doi:10.1038/s41598-018-25089-1.

Andersen, L.M., Jerbi, K., Dalal, S.S., 2020. Can EEG and MEG detect signals from the human cerebellum? Neuroimage 215, 116817. doi:10.1016/j.neuroimage.2020.116817.

Baselice, F., Sorriso, A., Rucco, R., et al., 2019. Phase linearity measurement: a novel index for brain functional connectivity. IEEE Trans. Med. Imaging 38, 873-882. doi:10.1109/TMI.2018.2873423.

Botvinik-Nezer, R., Holzmeister, F., Camerer, C.F., et al., 2020. Variability in the analysis of a single neuroimaging dataset by many teams. Nature 582, 84-88. doi:10.1038/s41586-020-2314-9.

Braak, H., Braak, E., 1991. Neuropathological stageing of Alzheimer-related changes. Acta Neuropathol. (Berl) 82, 239-259. doi:10.1007/BF00308809.

Brookes, M.J., Hale, J.R., Zumer, J.M., et al., 2011. Measuring functional connectivity using MEG: methodology and comparison with fcMRI. Neuroimage 56, 1082-1104. doi:10.1016/j.neuroimage.2011.02.054

Brookes, M.J., Woolrich, M.W., Barnes, G.R., 2012. Measuring functional connectivity in MEG: a multivariate approach insensitive to linear source leakage. Published Online Firs. t doi:10.1016/j.neuroimage.2012.03.048.

Bullmore, E., Sporns, O., 2009. Complex brain networks: graph theoretical analysis of structural and functional systems. Nat. Rev. Neurosci. 10, 186-198. doi:10.1038/nrn2575.

Carlesimo, G.A., Caltagirone, C., Gainotti, G., 1996. The Mental Deterioration Battery: normative data, diagnostic reliability and qualitative analyses of cognitive impairment. The Group for the Standardization of the Mental Deterioration Battery. Eur. Neurol. 36, 378-384. doi:10.1159/000117297.
Colclough, G.L., Woolrich, M.W., Tewarie, P.K., et al., 2016. How reliable are MEG resting-state connectivity metrics? Neuroimage 138, 284-293. doi:10.1016/j.neuroimage.2016.05.070.

Contreras, J.A., Goñi, J., Risacher, S.L., et al., 2017. Cognitive complaints in older adults at risk for Alzheimer's disease are associated with altered resting-state networks. Alzheimers Dement. Diagn. Assess Dis. Monit. 6, 40-49.

Corbetta, M., Shulman, G.L., 2002. Control of goal-directed and stimulus-driven attention in the brain. Nat. Rev. Neurosci. 3, 201-215. doi:10.1038/nrn755.

de Cheveigné, A., Simon, J.Z., 2007. Denoising based on time-shift PCA. J. Neurosci. Methods 165, 297-305. doi:10.1016/J.JNEUMETH.2007.06.003.

Deco, G., Jirsa, V., McIntosh, A.R., et al., 2009. Key role of coupling, delay, and noise in resting brain fluctuations. Proc. Natl. Acad. Sci. U. S. A. 106, 10302-10307. doi:10.1073/pnas.0901831106.

Demuru, M., Gouw, A.A., Hillebrand, A., et al., 2017. Functional and effective whole brain connectivity using magnetoencephalography to identify monozygotic twin pairs. Sci. Rep. 7, 9685. doi:10.1038/s41598-017-10235-y.

de Pasquale, F., Della Penna, S., Snyder, A.Z., et al., 2012. A cortical core for dynamic integration of functional networks in the resting human brain. Neuron 74, 753-764. doi:10.1016/j.neuron.2012.03.031.

Engels, M.M.A., van der Flier, W.M., Stam, C.J., et al., 2017. Alzheimer's disease: the state of the art in resting-state magnetoencephalography. Clin. Neurophysiol. 128, 14261437. doi:10.1016/j.clinph.2017.05.012.

Engel, A.K., Gerloff, C., Hilgetag, C.C., et al., 2013. Review intrinsic coupling modes : multiscale interactions in ongoing brain activity. Neuron 80, 867-886. doi:10.1016/j.neuron.2013.09.038.

Engemann, D.A., Kozynets, O., Sabbagh, D., et al., 2020. Combining magnetoencephalography with magnetic resonance imaging enhances learning of surrogate-biomarkers. Elife 9, e54055. doi:10.7554/eLife.54055.

Fazekas F., Chawluk J.B., Alavi A. MR signal abnormalities at $1.5 \mathrm{~T}$ in Alzheimer's dementia and normal aging. 1987. doi:10.2214/ajr.149.2.351

Finn, E.S., Shen, X., Scheinost, D., et al., 2015. Functional connectome fingerprinting: identifying individuals using patterns of brain connectivity. Nat. Neurosci. 18, 1664 1671. doi: $10.1038 / \mathrm{nn} .4135$

Finn, E.S., Bandettini, P.A., 2021. Movie-watching outperforms rest for functional connectivity-based prediction of behavior. Neuroimage 235, 117963. doi:10.1016/j.neuroimage.2021.117963.

Fornito, A., Zalesky, A., Bullmore, E., 2016. Fundamentals of Brain Network Analysis. Academic Press.

Fornito, A., Zalesky, A., Breakspear, M., 2015. The connectomics of brain disorders. Nat. Rev. Neurosci. 16, 159-172.

Folstein, M.F., Folstein, S.E., McHugh, P.R., 1975. Mini-mental state”. A practical method for grading the cognitive state of patients for the clinician. J. Psychiatr. Res. 12, 189198. doi:10.1016/0022-3956(75)90026-6.

Frasson, P., Ghiretti, R., Catricalà, E., et al., 2011. Free and cued selective reminding test: an Italian normative study. Neurol. Sci. 32, 1057-1062. doi:10.1007/s10072-011-0607-3.

FreeSurfer, Fischl B., 2012. Neuroimage 62, 774-781.

Friston, K.J., 2011. Functional and effective connectivity: a review. Brain Connect 1, 13 36. doi:10.1089/brain.2011.0008.

Hartoyo, A., Cadusch, P.J., Liley, D.T.J., et al., 2019. Parameter estimation and identifiability in a neural population model for electro-cortical activity. PLoS Comput. Biol. 15, e1006694. doi:10.1371/journal.pcbi.1006694.

Hillebrand, A., Tewarie, P., van Dellen, E., et al., March 2016. Direction of information flow in large-scale resting-state networks is frequency-dependent. Proc. Natl. Acad. Sci. U S A Published Online First doi:10.1073/pnas.1515657113.

Ito, M., 2006. Cerebellar circuitry as a neuronal machine. Prog. Neurobiol. 78, 272-303. doi:10.1016/j.pneurobio.2006.02.006.

Jacini, F., Sorrentino, P., Lardone, A., et al., 2018. Amnestic Mild Cognitive Impairment Is Associated With Frequency-Specific Brain Network Alterations in Temporal Poles. Front. Aging Neurosci. 10, 400. doi:10.3389/fnagi.2018.00400.

Koch, G.G., 2014. Intraclass Correlation Coefficient. Wiley StatsRef Stat Ref Online.

Lardone, A., Liparoti, M., Sorrentino, P., et al., 2018. Mindfulness meditation is related to long-lasting changes in hippocampal functional topology during resting state: a magnetoencephalography study. Neural Plast. 2018.

López, M.E., Bruña, R., Aurtenetxe, S., et al., 2014. Alpha-band hypersynchronization in progressive mild cognitive impairment: a magnetoencephalography study. J. Neurosci. Off. J. Soc. Neurosci. 34, 14551-14559. doi:10.1523/JNEUROSCI.0964-14.2014.

Maestú, F., Yubero, R., Moratti, S., et al., 2011. Brain activity patterns in stable and progressive mild cognitive impairment during working memory as evidenced by magnetoencephalography. J. Clin. Neurophysiol. Off. Publ. Am. Electroencephalogr. Soc. 28, 202-209. doi:10.1097/WNP.0b013e3182121743.

Miranda-Dominguez, O., Mills, B.D., Carpenter, S.D., et al., 2014. Connectotyping: model Based Fingerprinting of the Functional Connectome. PLoS ONE 9, e111048. doi:10.1371/journal.pone.0111048.

Moretti, D.V., Miniussi, C., Frisoni, G.B., et al., 2007. Hippocampal atrophy and EEG markers in subjects with mild cognitive impairment. Clin. Neurophysiol. Off. J. Int. Fed. Clin. Neurophysiol. 118, 2716-2729. doi:10.1016/j.clinph.2007.09.059.

Müller, F., Niso, G., Samiee, S., et al., 2019. A thalamocortical pathway for fast rerouting of tactile information to occipital cortex in congenital blindness. Nat. Commun. 10 5154. doi:10.1038/s41467-019-13173-7.

Noble, S., Scheinost, D., Constable, R.T., 2019. A decade of test-retest reliability of functional connectivity: a systematic review and meta-analysis. Neuroimage 203, 116157. doi:10.1016/j.neuroimage.2019.116157. 
Nolte, G., 2003. The magnetic lead field theorem in the quasi-static approximation and its use for magnetoencephalography forward calculation in realistic volume conductors. Phys. Med. Biol. 48, 3637-3652. doi:10.1088/0031-9155/48/22/002

Oostenveld, R., Fries, P., Maris, E., et al., 2011. FieldTrip: open Source Software for Advanced Analysis of MEG, EEG, and Invasive Electrophysiological Data, FieldTrip: open Source Software for Advanced Analysis of MEG, EEG, and Invasive Electrophysiological Data. Comput. Intell. Neurosci. Published Online First doi:10.1155/2011/156869, $10.1155 / 2011 / 156869$

Petersen, R., Smith, G., Waring, S., 1999. Mild cognitive impairment: clinical characterization and outcome. Arch. Neurol. 56, 303-309. doi:10.1001/archneur.56.3.303.

Pizzo, F., Roehri, N., Medina Villalon, S., et al., 2019. Deep brain activities can be detected with magnetoencephalography. Nat. Commun. 10, 971. doi:10.1038/s41467-019-08665-5.

Rombetto, S., Granata, C., Vettoliere, A., et al., 2014. Multichannel System Based on a High Sensitivity Superconductive Sensor for Magnetoencephalography. Sensors 14, 12114-12126. doi:10.3390/s140712114.

Ronga, B., Pellegrino, L., Loré, E., et al., 2004. The Frontal Assessment Battery (FAB): normative data from an Italian sample and performances of patients with Alzheimer's disease and frontotemporal dementia. Funct. Neurol.

Rucco, R., Liparoti, M., Jacini, F., et al., 2019. Mutations in the SPAST gene causing hereditary spastic paraplegia are related to global topological alterations in brain functional networks. Neurol. Sci. 40, 979-984.

Rucco, R., Baselice, F., Ambrosanio, M., et al., 2020. Brain connectivity study by multichannel system based on superconducting quantum magnetic sensors. Eng. Res. Express 2, 015038. doi:10.1088/2631-8695/ab7869.

Sadasivan, P.K., 1996. Narayana Dutt D. SVD based technique for noise reduction in electroencephalographic signals. Signal Process. 55, 179-189.

Samuelsson, J.G., Sundaram, P., Khan, S., et al., 2020. Detectability of cerebellar activity with magnetoencephalography and electroencephalography. Hum. Brain Mapp. 41, 2357-2372. doi:10.1002/hbm.24951.
Shen, X., Finn, E.S., Scheinost, D., et al., 2017. Using connectome-based predictive modeling to predict individual behavior from brain connectivity. Nat. Protoc. 12, 506-518.

Sica, C. Ghisi, M., 2007. The Italian versions of the Beck Anxiety Inventory and the Beck Depression Inventory-II: psychometric properties and discriminant power. Lead-Edge Psychol. Tests Test Res. 27-50.

Sorrentino, P., Rucco, R., Baselice, F., et al., 2019. Extensive functional repertoire underpins complex behaviours: insights from Parkinson's disease. Cold Spring Harbor Lab. doi:10.1101/823849.

Stam, C.J., Nolte, G., Daffertshofer, A., 2007. Phase lag index: assessment of functional connectivity from multi channel EEG and MEG with diminished bias from common sources. Hum. Brain Mapp. 28, 1178-1193. doi:10.1002/hbm.20346.

Svaldi, D.O., Goñi, J., Sanjay, A.B., et al., 2018. Towards subject and diagnostic identifiability in the alzheimer's disease spectrum based on functional connectomes. In Graphs in Biomedical Image Analysis and Integrating Medical Imaging and Non-Imaging Modalities. Springer, pp. 74-82.

Tzourio-Mazoyer, N., Landeau, B., Papathanassiou, D., et al., 2002. Automated anatomical labeling of activations in SPM using a macroscopic anatomical parcellation of the MNI MRI single-subject brain. Neuroimage 15, 273-289. doi:10.1006/nimg.2001.0978.

Van Veen, B.D., Van Drongelen, W., Yuchtman, M., et al., 1997. Localization of Brain Electrical Activity via Linearly Constrained Minimum Variance Spatial Filtering. IEEE Trans. Biomed. Eng. 44.

Vinck, M., Oostenveld, R., Van Wingerden, M., et al., 2011. An improved index of phase-synchronization for electrophysiological data in the presence of volume-conduction, noise and sample-size bias. Neuroimage 55, 1548-1565. doi:10.1016/j.neuroimage.2011.01.055.

Varoquaux, G., Raamana, P.R., Engemann, D.A., et al., 2017. Assessing and tuning brain decoders: cross-validation, caveats, and guidelines. Neuroimage 145, 166-179.

Varoquaux, G., 2018. Cross-validation failure: small sample sizes lead to large error bars. Neuroimage 180, 68-77. 